\title{
Artificial anterior chamber for the growing of membranes on lens implants *
}

\author{
J. Reimer Wolter ${ }^{1 * *}$ and Steven L. Kunkel ${ }^{2}$ \\ Departments of Ophthalmology and Pathology ${ }^{1}$ and Department of Pathology ${ }^{2}$, University of Michigan Medical Center, \\ Ann Arbor, Michigan 48109, USA
}

\begin{abstract}
Intraocular lens implants made of glass and plastic were placed into the lumen of short pieces of clear plastic tubing. These were incompletely closed at both ends with nylon sutures, filled with Healon, and used experimentally as artificial anterior chambers for implantation into the peritoneal space of mice. After 7 days macrophages had been attracted into the chambers, become sessile on the implants, and formed a continuous membrane on their surface. The concept of artificial anterior chambers in experimental and future clinical applications is discussed.
\end{abstract}

\section{Introduction}

Earlier studies [1-6] have indicated that the inner eye reacts to a newly introduced lens implant by mobilizing free-moving macrophages. These are attracted to the surface of the foreign substance, become adherent, and change into sessile epithelioid cells, fibroblastlike cells, or giant cells. They also are involved in the formation of a separating proteinaceous film that develops with time into a capsulelike formation continuously covering all parts of the implant. From a successful implant the free-moving macrophages and most of the giant cells disappear with time. Relatively few fibroblastlike cells remain permanently and maintain the capsule. The formation of this type of reactive membrane on lens implants is considered beneficial. They are optically clear and prevent direct contact of the fluid and tissue structures of the inner eye with the foreign substances of the implants.

The process of the reactive formation of continuous and optically clear membranes on the surface of lens implants is not always uncomplicated. For example, the membrane may become thick and scarlike. This can result in optical obstruction as well as in obliteration of adjacent spaces. Vitreous formation on the surface of implants has been seen to interfere with the successful structure of a proper separating membrane. The impression is that implants without a continuous membrane are not tolerated well, because continuing uveitis and damage to the corneal endothelium

\footnotetext{
* Supported by The Research to Prevent Blindness, Inc. New York, N.Y. USA

** Corresponding author
}

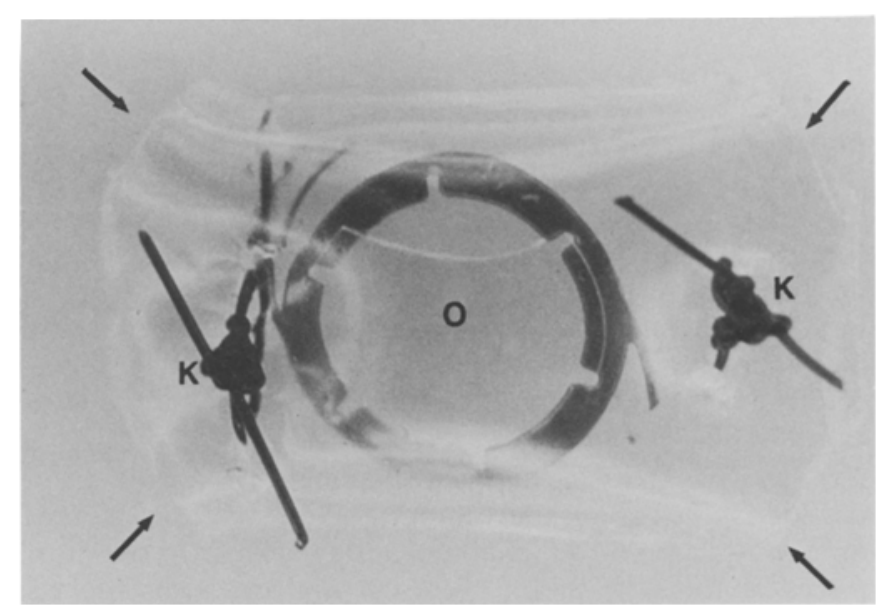

Fig. 1. One of the artificial anterior chambers containing a glass intraocular lens implant before implantation. The optic portion of the implant $(O)$ is clearly seen within the plastic tubing (arrows) flattened by knotted nylon sutures $(K)$. The haptics of the implant are cut short. Photograph $\times 15$

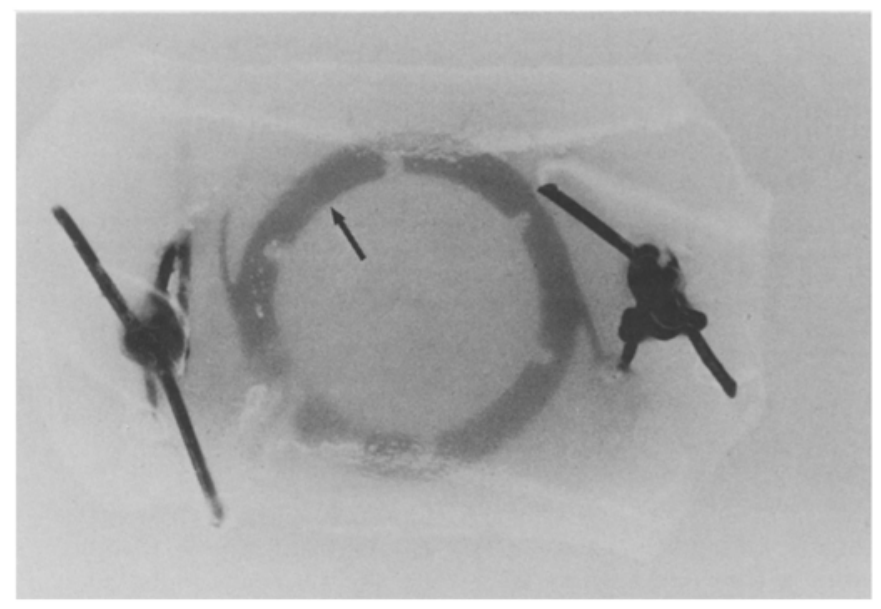

Fig. 2. After 7 days' implantation in the peritoneal space of a mouse. The chamber contains a milky fluid and all parts are covered by a grayish layer. The rim of the glass implant is visible in the chamber (arrow). Photograph $\times 15$ 


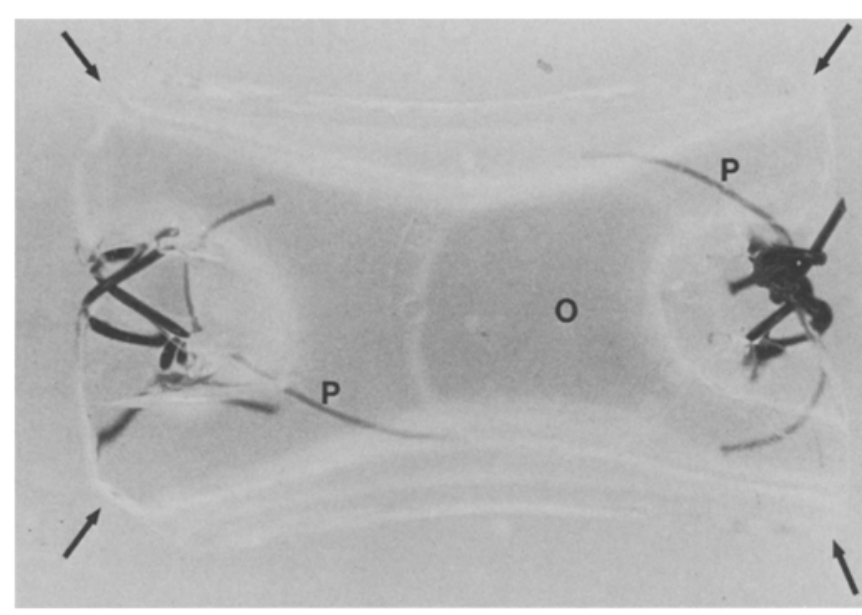

Fig. 3. The other artificial anterior chamber (arrows) containing a polymethylmethacrylate implant $(O)$ with blue Prolene haptics $(P)$ before implantation. Photograph $\times 15$

have been seen to be pronounced in association with the presence of naked surfaces of implants.

Initially, the concept of the artificial anterior chamber for the study of membranes on implants under experimental conditions is presented in this paper. In the future we think that the use of artificial anterior chambers might be one way to grow clear separating membranes of the patients own monocytes on the surface of his or her lens implant, before this is placed in the eye.

Experiment. Artificial anterior chambers were built from the kind of clear plastic tubing used in operating rooms or laboratories and supplied in sterile packages. Two short pieces of this soft tubing were cut and one lens implant of glass and another of polymethylmethacrylate with Prolene haptics was placed in the lumen of the pieces of tubing. ${ }^{1}$ The tubing was flattened out in this process and both ends were closed in a flattened position by nylon sutures (Figs. 1 and 2). Thus the lumen of the tubing was closed off at both ends and a central chamber containing the implants was created. In the region of the tied sutures the opposite walls of the tubing were tightly touching. However, there were four narrow openings into the center of the chamber preserved on both sides, next ot the nylon sutures. The size of the pieces of tubing made into artificial anterior chambers was approximately $9 \times 6 \times 4 \mathrm{~mm}$.

Under sterile conditions these artificial anterior chambers were filled with Healon (sodium hyaluronate) and placed into the peritoneal space of two black laboratory mice. The mice measured $50 \mathrm{~mm}$ in length without tails.

\footnotetext{
1 This is to thank IOLAB Corporation of Covina, California and Lynell Medical Technology, Inc., of New York, N.Y. for supplying the lens implants used for this study
}

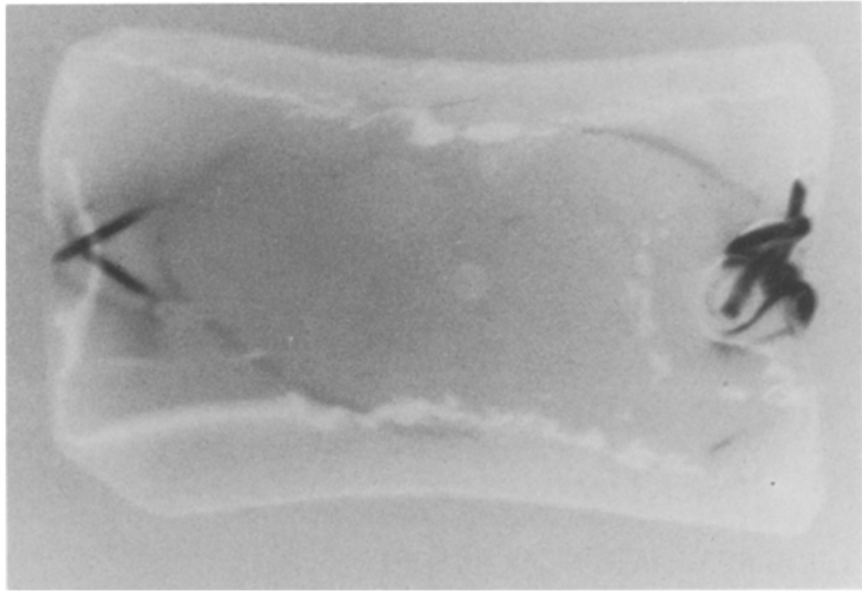

Fig. 4. The artificial anterior chamber seen in Fig. 3 after 7 days' implantation in the peritoneal space of a mouse. The chamber is covered with a gray layer and contains a milky fluid. Photography $\times 15$

The implantation of the flat pieces of plastic tubing was a little tight due to the large size of the tubes in relation to the mice. However, the mice remained active, ate, and did not appear sick or in any way incapacitated throughout the week of the experiment. The mice survived the operation without any difficulties. After 7 days the mice were killed. The artificial anterior chambers were found in the peritoneal space without firm adhesions and they could be removed without difficulty. Both pieces of tubing were covered with a smooth layer of slightly yellowish material on the outside. Both contained an exudatelike yellowish fluid in their lumen (Figs. 3 and 4). The artificial anterior chambers were immediately placed into $10 \%$ buffered formalin for a day of fixation.

The specimens were washed as a whole and then the pieces of tubing were opened with scissors on one side. A yellowish exudatelike layer of mushy substance was attached to the implants as well as to all the surfaces of the plastic tubing. The implants together with the attached layer were removed without difficulty. The lens implant cytology technique was used to stain the layers on both implants with hematoxylin-eosin [3].

The glass implant had a thick covering of firmly attached cell layers. These resembled free-moving macrophages and early stages of fibroblastlike cells, mostly of bipolar shape. However, technical difficulties were encountered in the dehydrating and embedding process, after the staining was completed and a preliminary inspection of the specimen had been carried out under the microscope. The plastic implant from the other piece of tubing, however could be dehydrated, embedded, and photographed properly. The polymethylmethacrylate optic portion of the implant was, in the first place, tightly surrounded by a thin eosinophilic film (Figs. 5-7).

Fig. 5. Low-power view of the plastic implant removed from the artificial anterior chamber seen in Fig. 4. Its optic part $(O)$ is covered with a thin and regular layer of a slightly eosinophilic proteinaceous substance. Dark staining areas of fibrinous exudate are seen in a somewhat geographical arrangement on parts of the optics. The Prolene haptics $(P)$ show one thick accumulation of cells $($ arrow). Lens implant cytology technique, $\mathrm{H}+\mathrm{E}$ stain, photomicrograph $\times 25$

Fig. 6. Portion of the optics of the plastic lens implant with its thin homogenous capsulelike layer $(O)$ and loose additional accumulations of fibrin on its surface (arrows). Lens implant cytology technique, $\mathrm{H}+\mathrm{E}$ stain, photomicrograph $\times 100$ 


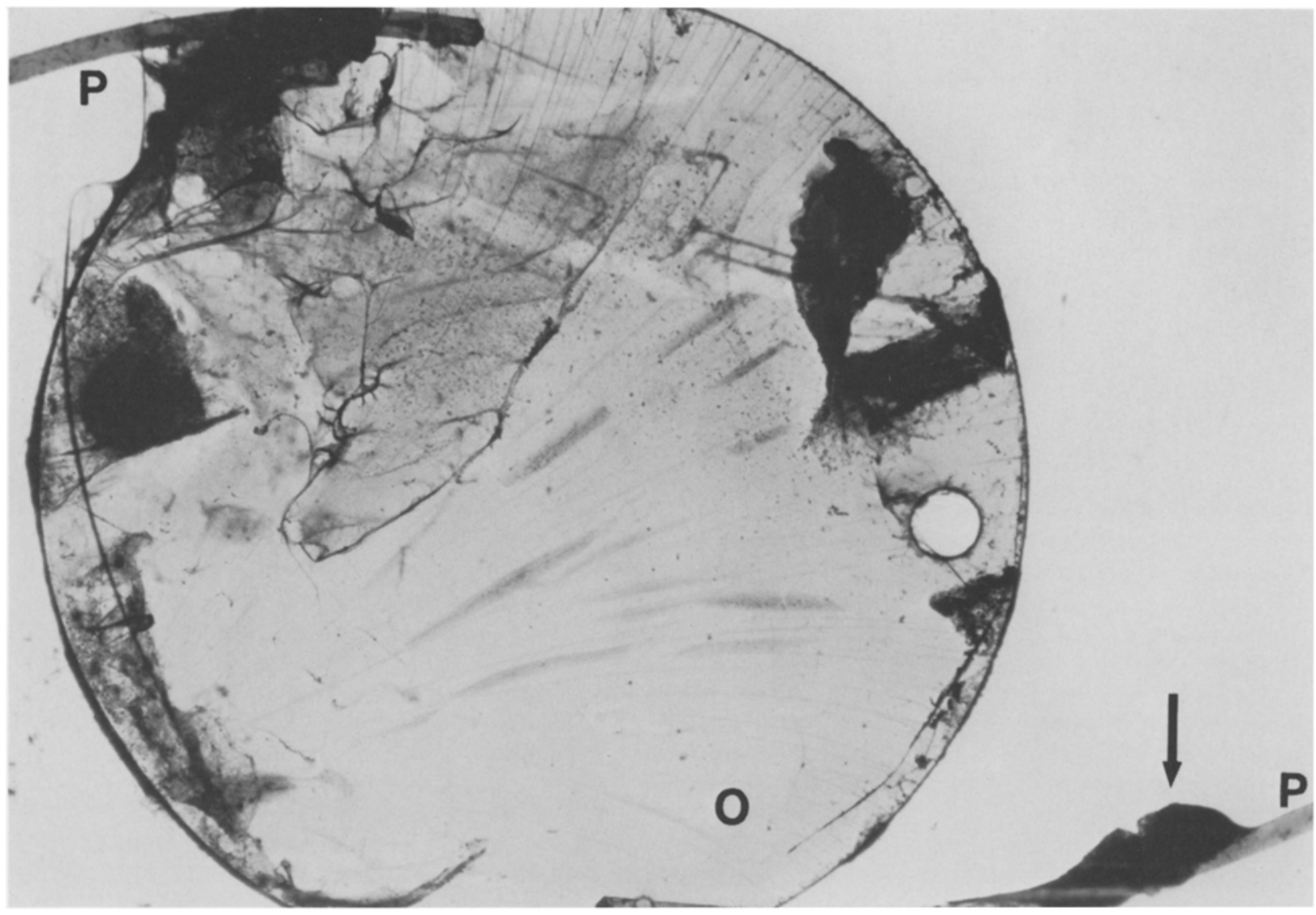

Fig. 5

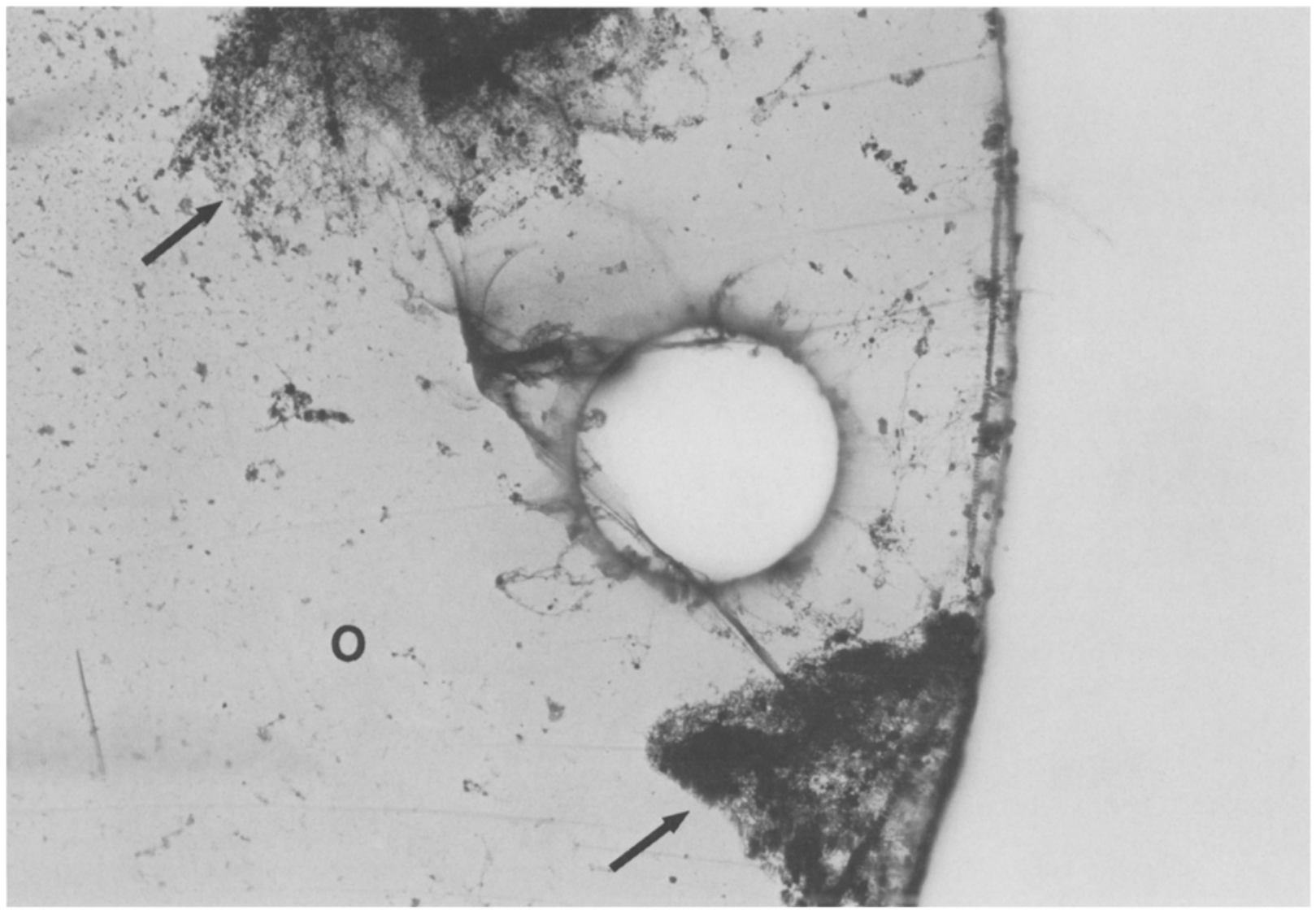

Fig. 6 


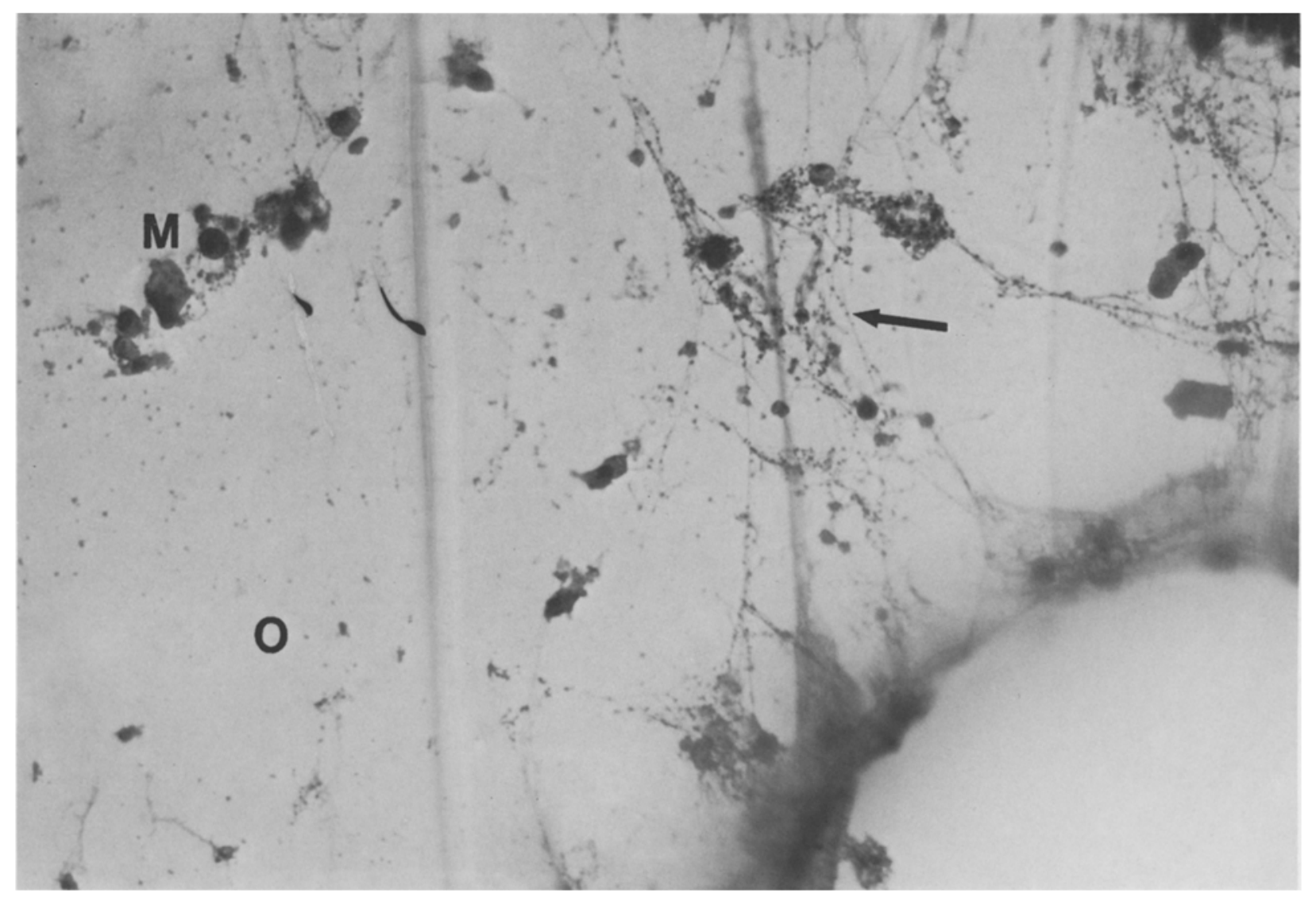

Fig. 7

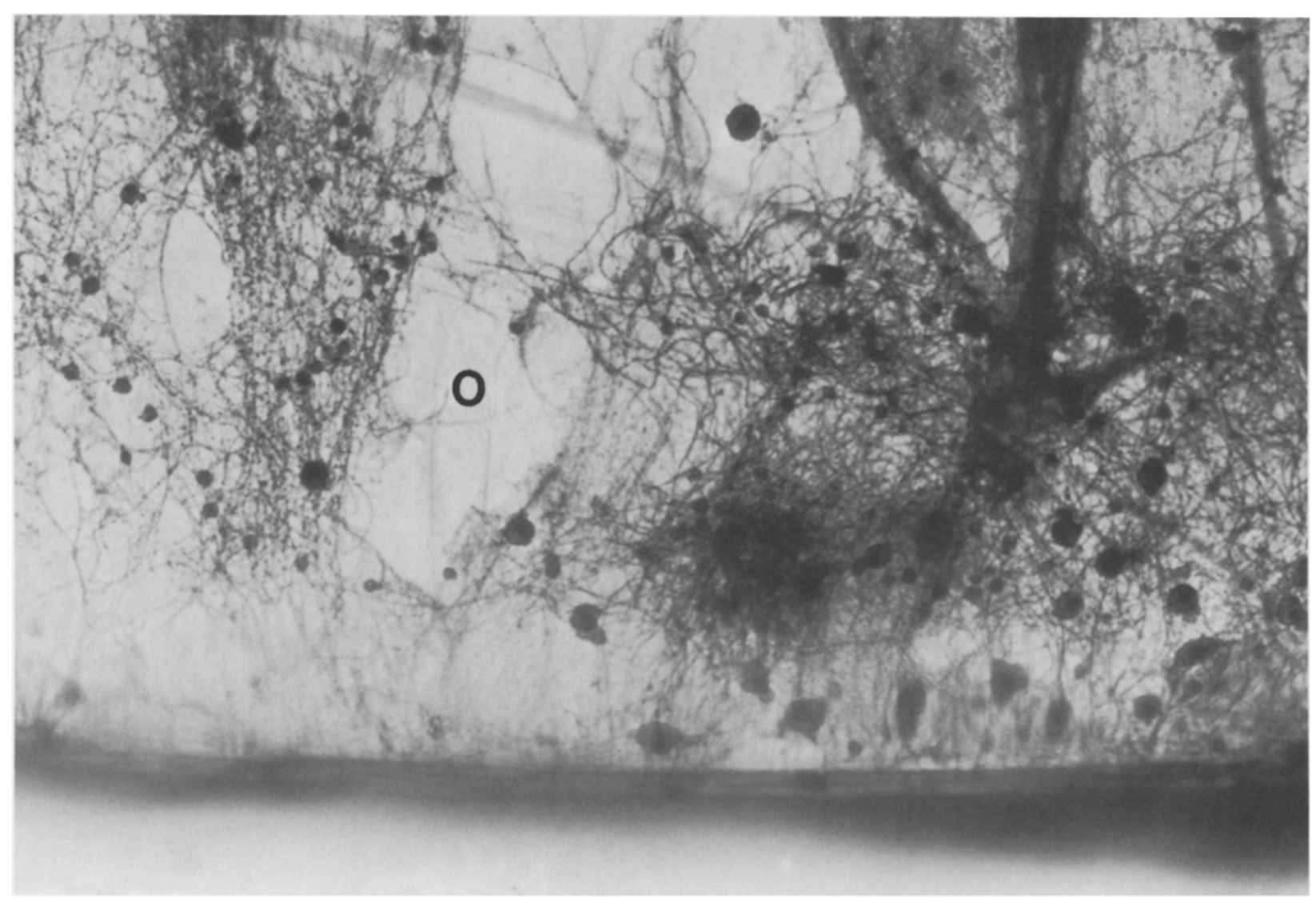




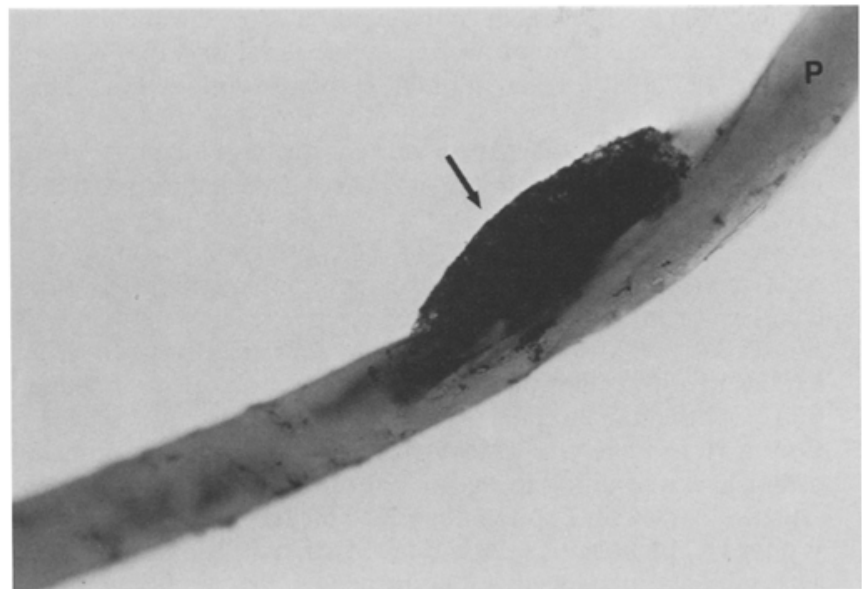

Fig. 9. High-power view of one of the Prolene haptics $(P)$ of the lens implant seen in Fig. 4 with single macrophages rather evenly distributed all over its surface and an additional nodular accumulation of densely arranged macrophages supported by a fibrin framework (arrow). Lens implant cytology technique, $\mathrm{H}+\mathrm{E}$ stain, photomicrograph $\times 100$

This was of rather homogenous composition and regular structure and contained macrophages in an irregular distribution (Figs. 6 and 7). Another less regular and much looser layer of fibrinous exudate containing more macrophages and occasional polymorphnuclear leukocytes (PMNs) was found on top of the homogenous thin layer on the surface of the implant (Figs. 5-8). The loose network of fibrin had clearly filled some of the space around the implant in the tubing and interconnected the reactive layers of the wall of this tubing and the surface of the implant. The most dense and cellular accumulations of macrophages were seen, in addition to diffusely distributed macrophages, on the Prolene haptics of this implant and presented as rounded bunches of cells with a fibrinous framework (Fig. 9).

\section{Discussion}

This first attempt to create an artificial anterior chamber for the growth of cellular membranes on lens implants in the peritoneal space of the mouse has been quite successful. Just like the anterior chamber of the eye, this artificial anterior chamber was fluid-filled. Healon was used for this purpose with the aim of avoiding the loss of a less viscous fluid from the chamber during the implantation process. Healon has been found not to interfere with the orderly formation of membranes on lens implants by peritoneal mouse macrophages under tissue culture conditions in a recently reported experiment by Wolter and Kunkel, which has been submitted for publication. The peritoneal macrophages were attracted by the implants in both artificial anterior chambers and found their way into their lumen through the narrow openings on both sides of the sutures. They adhered to the implants and within the 7 days of the experiment settled on the surface of the implants. This process was associated with the development of a filmlike, homogeneous, and slightly eosinophilic membrane of a proteinaceous substance all over the surface of both implants. It is suspected that the macrophages produced these membranes, but definite proof of this is not as yet available. On the glass implant fibroblastlike cells were seen in addition to common macrophages, but technical problems with its embedding prevented photographic documentation.

The thicker, looser, and more patchy reaction containing fibrin and PMNs superficially attached to the implants probably was part of the more acute inflammatory response elicited by the plastic tubing. This kind of a reaction was found all over the outside and the inside of both pieces of plastic tubing. Of all the plastic materials that we have studied, polymethylmethacrylate causes the least inflammatory reaction, when it is implanted in tissues or tissue fluids. This observation is supported by clinical findings which also show that polymethylmethacrylate is relatively very well tolerated. Supramid and Prolene are both known to elicit more of a cellular response, as well as a thicker proteinaceous capsule, when these substances are used for lens implants [6]. In the present experiment it was observed that the Prolene haptics had much more massive accumulations of cells than the polymethylmethacrylate optics. In comparison to all three of these types of plastics commonly utilized for lens implants, the tubing used for the artificial anterior chamber in the present experiment caused a much more severe reaction with thicker layers of cells, fibrinous exudate, and some PMNs all over its surface.

The idea of a fluid-filled artificial anterior chamber for the growing of cells and separating membranes on lens implants will be pursued. Since this first and rather crude model has shown such promise, a better chamber will be built, and obviously this has to be completely composed of polymethylmethacrylate in all parts. Other fluids for filling the chamber and new implantation sites in larger animals will be investigated with the aim of creating a situation resembling the inner eye as far as possible.

We foresee a time when intraocular lens implants, within a well designed artificial anterior chamber, will be temporarily placed somewhere in the organism of a patient who will need cataract surgery. The idea would be that all the biochemical and cellular interactions between the patient's phagocyte system and the foreign substance of the implant take place in the safe surroundings of the artificial anterior chamber, and that a permanent separating capsule is created before that implant is placed in its final place in the eye. The haptics could extend out of the fluid-filled artificial anterior chambers for the growth of fibrous attachments. These would be excised at the end of the period to fit an area of the wall of the inner eye for firm attachment. Tissue engineering in the truest sense would thus be used for the separation of the main body, as well as for the fixation of lens implants in the eye. Possible sites for the experimental implantation of artificial anterior

Fig. 7. Higher power of the fibrin network (arrow) on the surface of the homogeneous capsule with macrophages ( $M$ ) on the optic portion $(O)$ of the plastic implant seen in Fig. 4. Lens implant cytology technique, $\mathrm{H}+\mathrm{E}$ stain, photomicrograph $\times 250$

Fig. 8. Border of the plastic implant seen in Fig. 4. The fibrin network on its optic portion $(O)$ exhibits larger macrophages as well as small PMNs, Lens implant cytology technique, $\mathrm{H}+\mathrm{E}$ stain, photomicrograph $\times 250$ 
chambers are locations deep in the skin - possibly in the arm pits or on the side of the chest - in the peritoneal space, under the periosteum, or perhaps even in the bone marrow. The latter site has special appeal because it is the primary source of the macrophages. To obtain monocytes from the patient's blood and to grow membranes on lens implants in tissue culture in another possibility, but our experiences with mouse macrophages in tissue culture indicate that continuous supply of new macrophages over a relatively long time is necessary for the formation of useful membranes. The experimental path towards these goals looks open and quite inviting. However, much basic work needs to be done in animal experiments and much new insight will have to be gained before the dream of a lens implant with a pregrown separating membrane in a human eye can become true.

\section{References}

1. Wolter JR (1982) Cell life on the surface of lens implants. Graefe's Arch Clin Exp Ophthalmol 218:244-249
2. Wolter JR (1982) Foreign body giant cells on intraocular lens implants. Graefe's Arch Clin Exp Ophthalmol 219:103-111

3. Wolter JR (1982) Lens implant cytology. Ophthalmic Surg 13:726-732

4. Wolter JR (1983) Reactive membrane on lens implant: three month after implantation. Graefe's Arch Clin Exp Ophthalmol $220: 53-57$

5. Wolter JR (1983) Morphology of the capsulelike portion of the reactive membranes on intraocular lens implants. Graefe's Arch Clin Exp Ophthalmol 220:110-116

6. Wolter JR (1983) Foreign body giant cells selectively covering haptics of intraocular lens implants: indicators of poor toleration? Ophthalmic Surg (in press)

7. Wolter JR, Kunkel SL (1983) Abdominal implantation of intraocular lenses resulting in the formation of reactive membranes. Graefe's Arch Clin Exp Ophthalmol 220:215-222

8. Wolter JR, Lichter PR (1983) Fibroblastlike cells on intraocular lens implants: phagocytizing erythrocytes. Br J Ophthalmol (in print)

Received May 20, 1983 